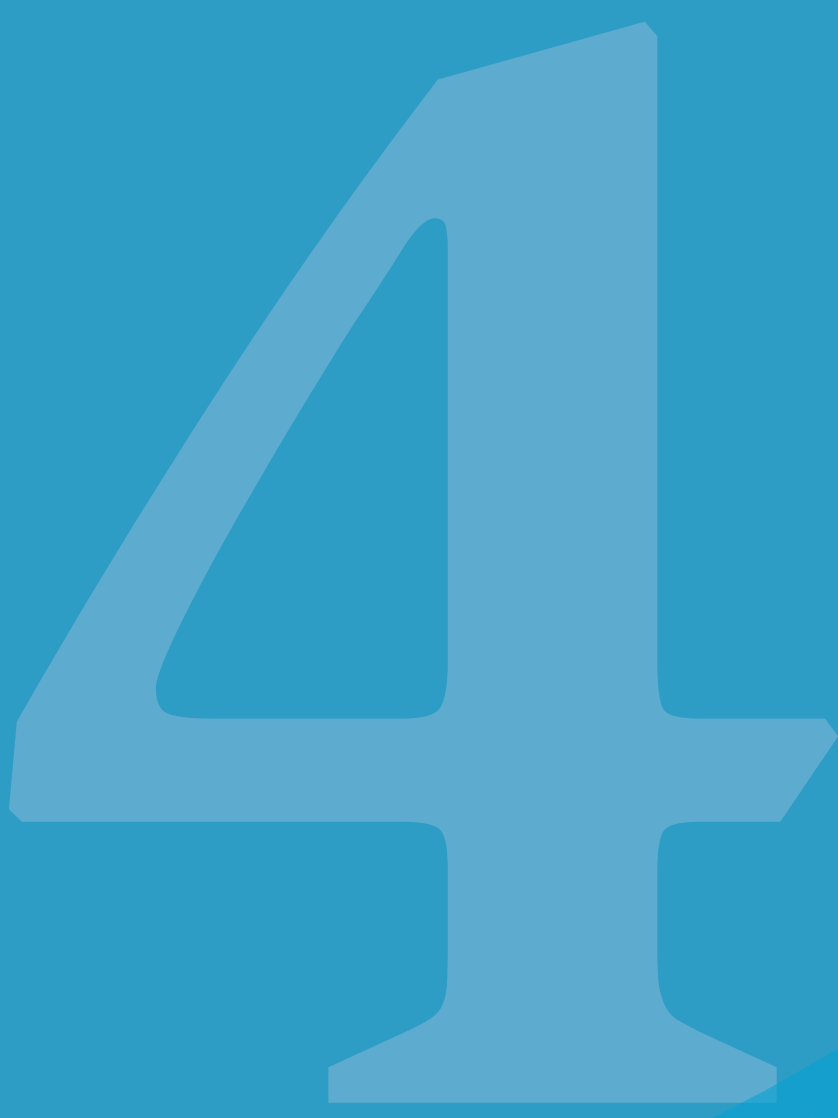


Capítulo 4

\section{Pertinencia de la formación para la
supervisión clínica formación para la
supervisión clínica}

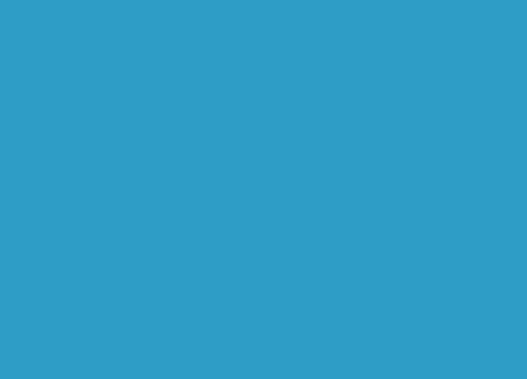

西
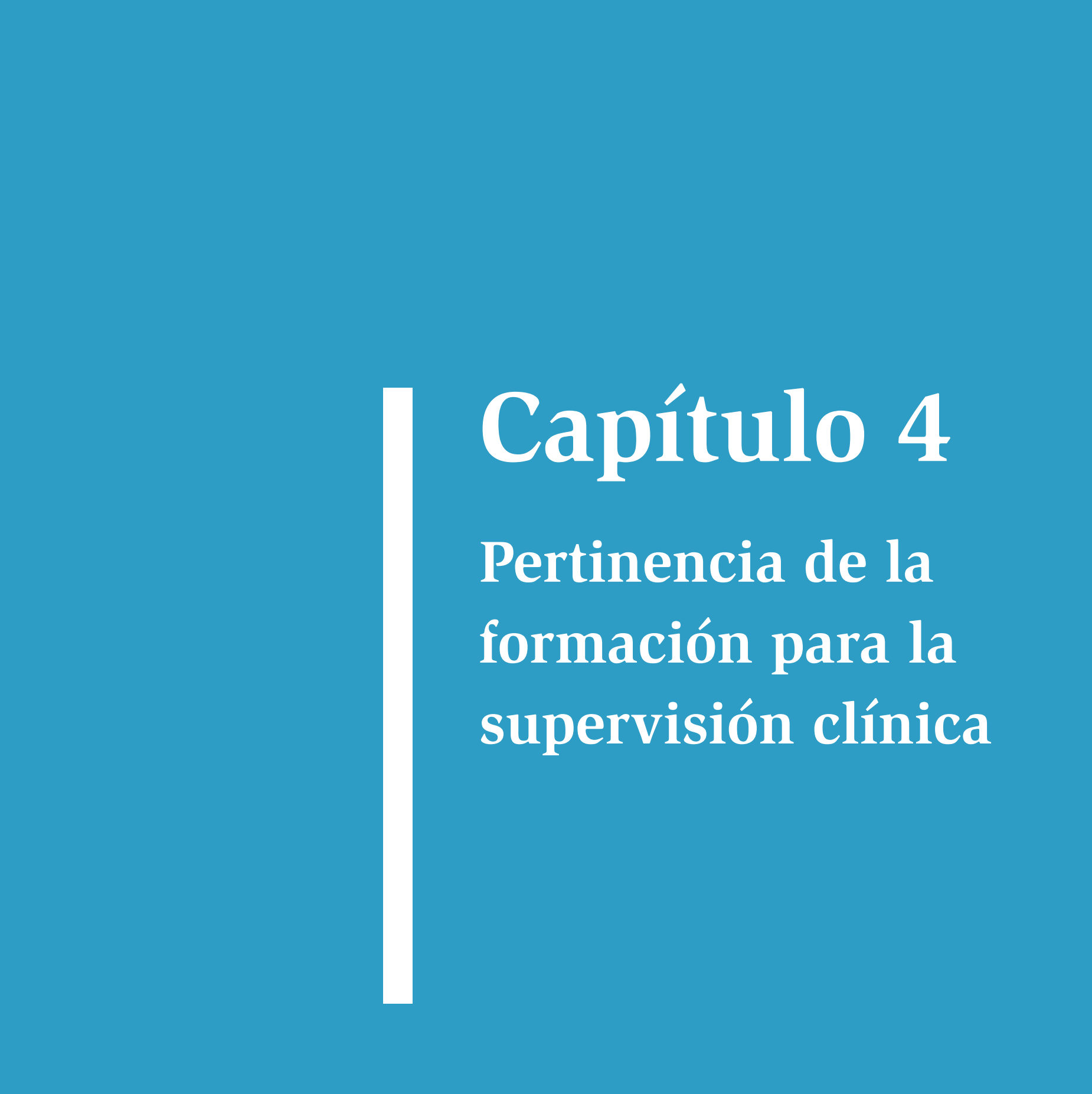



\section{Capítullo 4}

\section{Pertinencia de la formación para la supervisión clínica}

I Mónica Novoa Gómez, Ph.D y Óscar Alejandro Córdoba, Mg

\section{Resumen}

Diversos autores han definido la supervisión clínica como un momento esencial para el desarrollo de las habilidades necesarias para la psicoterapia, puesto que permite consolidar aprendizajes académicos, actitudes, conocimientos y habilidades necesarias para la profesión (Falender, 2014a; Martino et al., 2016; Novoa-Gómez, Córdoba-Salgado, Peñuela et al., en revisión) para mejorar la calidad de la atención clínica (Reiser y Milne, 2014). Es una modalidad pedagógica en la que el estudiante debe articular la teoría y la intervención para fortalecer sus competencias tanto terapéuticas como de investigación (Ballesteros et al., en revisión), en el que la enseñanza de las habilidades clínicas implica que el supervisor explore y evalúe las observaciones, sentimientos y pensamientos de los supervisados (Ballesteros et al., en revisión; Tomlin, Eeatherston y Pavkov, 2014; Watkins y Scaturo, 2013).

La supervisión clínica ha recibido mayor atención y se ha considerado una especialidad pedagógica y profesional (American Psychological Association -APA2015; Borders et al., 2014) con lineamientos, modelos y guías acerca de la mejor manera de desarrollarla, con base en la investigación empírica (e. g., Bernard y Goodyear, 2014; Ellis et al., 2014; Falender y Shafranske, 2014; Goodyear y Rodolfa, 2012; Newman, 2013). Diversos autores han desarrollado diferentes modelos de 
supervisión (Boswell, Nelson, Nordberg, Mcaleavey y Castonguay, 2010; Holt et al., 2015; Myers y Sweeney, 2008; Plakun, Sudak y Goldberg, 2009), algunos de los que tienen mayor soporte empírico son el modelo basado en la functional analytic psychotherapy (FAP) (Callaghan, 2006a, 2006b; Kohlenberg y Tsai, 1991), competency based clinical supervision (Falender, 2014b), cube model for competency development (Rodolfa et al., 2013) y el evidence based clinical supervision (Milne, 2009). Además, se han desarrollado diferentes instrumentos para medir la efectividad de la supervisión como la escala de terapia cognitiva (Newman, 2010), teacher's pet (Milne, 2009), el enhacing assessment of common therapeutic factors (Kohrt et al., 2015) y the supervision outcome scale's (Tsong y Goodyear, 2014).

Los modelos de supervisión establecen una serie de objetivos referentes a la relación entre el supervisor y el supervisado que se basan en la educación y el entrenamiento, la gestión, desarrollo y la evaluación del trabajo del último (Milne, 2009; Rodolfa et al., 2013). De tal forma que el supervisado pueda recibir apoyo en caso de necesitarlo (Callaghan, 2006a; Milne, 2009), recibir entrenamiento y ser evaluado en el desarrollo de unas competencias específicas (APA, 2015; Callahan et al., 2017; Falender, 2014b; Rodolfa et al., 2013, Rosenbluth, Cruzat-Mandich y Ugarte, 2016), a la vez que se busca el bienestar y protección del consultante (APA, 2015; BastidasBilbao y Velásquez, 2016; Bernard y Goodyear, 2014; Callaghan, 2006a; Falender, 2014b; Milne, 2009; Newman, 2010; Reiser y Milne, 2014). Falender et al. (2004) plantean las competencias como un uso habitual y juicioso de comunicación, conocimiento, habilidades técnicas, razonamiento clínico, emociones, valores y reflexiones que se utilizan en la práctica y, más adelante, resume como conocimientos, habilidades y actitudes (Falender, 2014b) que requieren de retroalimentación sistemática para su desarrollo (APA, 2015; Carmel, Villatte, Rosenthal, Chalker y Comtois, 2016; Newman, 2010). Rodolfa et al. (2005) plantean que existen las competencias fundacionales, relacionadas con el profesionalismo que implica actuar acorde con los estándares éticos, colaboración interdisciplinar, habilidades de relaciones interpersonales y capacidad de autoconocimientos, entre otras. Las competencias funcionales, por su parte, están relacionadas con habilidades específicas y conocimientos, tales como realizar un diagnóstico, estructurar una sesión, conceptualizar el caso, entre otros (Genuchi et al., 2015; Newman, 2010). Por otro lado, se debe tener en cuenta el proceso de la supervisión. Se ha conceptualizado de diversas formas el proceso de la supervisión. Milne (2018) plantea los siguientes elementos como parte de la supervisión: a) evaluación inicial, b) desarrollo de estrategias de evaluación para la adquisición de dichas competencias, c) selección 
de contenido, d) selección de estrategias y material para el entrenamiento, e) realizar las actividades de aprendizaje, f) evaluar y g) retroalimentar el contenido.

Un factor relevante para este proceso está relacionado con el apoyo institucional y la forma de impartir la supervisión. Existen diferentes modalidades para impartir la supervisión: directa y sincrónica, como la coterapia que es una forma ideal de supervisión (Falke, Lawson, Pandit y Patrick, 2015). Está la directa y asincrónica cuando se realiza sin que el supervisor esté presente físicamente en la sesión terapéutica del supervisado, pero está disponible (Whalen y Wendel, 2011) y a través de videos o grabaciones de audio. Finalmente, indirecta y asincrónica cuando la supervisión no se da al mismo tiempo que la sesión y se basa en el reporte verbal o escrito de lo ocurrido en sesión o los reportes escritos de lo que se hizo en la sesión (Borders et al., 2014; Callaghan, 2006a, 2006b; Falender, 2014b; Martino et al., 2016; Newman, 2010; Reiser y Milne, 2012).

Dada la necesidad de ofrecer una supervisión que permita el desarrollo de las funciones que se le han atribuido, ha surgido la preocupación de las competencias del supervisor para ejercer esta función. Para ello se han diseñado diversos manuales para la supervisión (Bernard y Goodyear, 2014; Falender y Shafrenske, 2007; Milne, 2018). Sin embargo, dentro de este grupo de manuales resalta un manual de entrenamiento a supervisores, desarrollado por Milne y Reiser (2017), en el cual se profundizará en este capítulo.

\section{Conceptualización de la supervisión}

Diversos autores han definido la supervisión clínica como un momento esencial para el desarrollo de las habilidades necesarias para la psicoterapia, puesto que permite consolidar aprendizajes académicos, actitudes, conocimientos y habilidades necesarias para la profesión (Falender, 2014a; Martino et al., 2016; NovoaGómez, Córdoba-Salgado, Peñuela et al., en revisión) para mejorar la calidad de la atención clínica (Reiser y Milne, 2014). Es una modalidad pedagógica en la que el estudiante debe articular la teoría y la intervención para fortalecer sus competencias tanto terapéuticas como de investigación, en el que la enseñanza de las habilidades clínicas implica que el supervisor explore y evalúe las observaciones, sentimientos y pensamientos de los supervisados (Tomlin, Weatherston y Pavkov, 2014; Watkins y Scaturo, 2013). 
La supervisión clínica ha recibido mayor atención y se ha considerado una especialidad pedagógica y profesional (American Psychological Association -APA-, 2015; Borders et al., 2014) con lineamientos, modelos y guías acerca de la mejor manera de desarrollarla, con base en la investigación empírica (e. g., Bernard y Goodyear, 2014; Ellis et al., 2014; Falender y Shafranske, 2014; Goodyear y Rodolfa 2012; Newman, 2013). Diversos autores han desarrollado diferentes modelos de supervisión (Boswell, Nelson, Nordberg, McAleavey y Castonguay, 2010; Holt et al., 2015; Myers y Sweeney, 2008; Plakun, Sudak y Goldberg, 2009); algunos de los que tienen mayor soporte empírico son el modelo basado en la Functional Analytic Psychotherapy (FAP) (Callaghan, 2006a y 2006b; Kohlenberg y Tsai, 1991), Competency Based Clinical Supervision (Falender, 2014b), Cube Model for Competency Development (Rodolfa et al., 2013) y el Evidence Based Clinical Supervision (Milne, 2009). Además, se han desarrollado diferentes instrumentos para medir la efectividad de la supervisión como la Escala de Terapia Cognitiva (Newman, 2010), Teacher's PET (Milne, 2009a), el Enhacing Assessment of Common Therapeutic Factors (Kohrt et al., 2015) y The Supervision Outcome Scale's (Tsong y Goodyear, 2014).

\section{las competencias}

fundacionales, relacionadas

con el profesionalismo

que implica actuar acorde

con los estándares éticos, colaboración interdisciplinar,

habilidades de relaciones interpersonales y capacidad de autoconocimientos, entre

otras. Las competencias

funcionales, por su parte,

están relacionadas con

habilidades específicas y

conocimientos.
Los modelos de supervisión establecen una serie de objetivos referentes a la relación entre el supervisor y el supervisado que se basan en la educación y el entrenamiento, la gestión, desarrollo y la evaluación del trabajo del último (Milne, 2009b; Rodolfa et. al., 2013). De tal forma que el supervisado pueda recibir apoyo en caso de necesitarlo (Callaghan, 2006a; Milne, 2009b), recibir entrenamiento y ser evaluado en el desarrollo de unas competencias específicas (APA, 2015; Callahan, Neumann, Cox y Ruggero, 2017; Falender, 2014b; Rodolfa et, al., 2013, Rosenbluth, Cruzat-Mandich y Ugarte, 2016), a la vez que se busca el bienestar y la protección del consultante (APA, 2015; BastidasBilbao y Velásquez, 2016; Bernard y Goodyear, 2014; Callaghan, 2006a; Falender, 2014b; Milne, 2009b; Newman, 2010; Reiser y Milne, 2014). Falender et al. (2004) plantean las competencias como un uso habitual y juicioso de comunicación, conocimiento, habilidades técnicas, razonamiento clínico, emociones, valores y reflexiones que se utilizan en la práctica y, más adelante, resume como conocimientos, habilidades y actitudes (Falender, 2014b) que requieren de 
retroalimentación sistemática para su desarrollo (APA, 2015; Carmel, Villatte, Rosenthal, Chalker y Comtois, 2016; Newman, 2010). Rodolfa et al. (2005) plantean que existen las competencias fundacionales, relacionadas con el profesionalismo que implica actuar acorde con los estándares éticos, colaboración interdisciplinar, habilidades de relaciones interpersonales y capacidad de autoconocimientos, entre otras. Las competencias funcionales, por su parte, están relacionadas con habilidades específicas y conocimientos, tales como realizar un diagnóstico, estructurar una sesión, conceptualizar el caso, entre otros (Genuchi, Rings, Germek y Cornish, 2015; Newman, 2010).

Por otro lado, debe tenerse en cuenta el proceso de la supervisión. Se ha conceptualizado de diversas formas el proceso de la supervisión. Milne (2018) plantea los siguientes elementos como parte de la supervisión: a) evaluación inicial, b) desarrollo de estrategias de evaluación para la adquisición de dichas competencias, c) selección de contenido, d) selección de estrategias y material para el entrenamiento, e) realizar las actividades de aprendizaje, f) evaluar y g) retroalimentar el contenido.

Un factor relevante para este proceso está relacionado con el apoyo institucional y la forma de impartir la supervisión. Existen diferentes modalidades para impartir la supervisión: directa y sincrónica, como la coterapia que es una forma ideal de supervisión (Falke, Lawson, Pandit y Patrick, 2015). Está la directa y asincrónica cuando se realiza sin que el supervisor esté presente físicamente en la sesión terapéutica del supervisado, pero está disponible (Whalen y Wendel, 2011) y mediante videos o grabaciones de audio. Finalmente, indirecta y asincrónica cuando la supervisión no se da al mismo tiempo que la sesión y se basa en el reporte verbal o escrito de lo ocurrido en la sesión o en los reportes escritos de lo que se hizo en ella (Borders et al., 2014; Callaghan, 2006a, 2006b; Falender, 2014b; Martino et al., 2016; Newman, 2010; Reiser y Milne, 2012).

Dada la necesidad de ofrecer una supervisión que permita el desarrollo de las funciones que se le han atribuido, ha surgido la preocupación de las competencias del supervisor para ejercer esta función. Para ello se han diseñado diversos manuales para la supervisión (Bernard y Goodyear, 2014; Falender y Shafranske, 2004; Milne 2018). Sin embargo, dentro de este grupo de manuales resalta un manual de entrenamiento a supervisores, desarrollado por Milne y Reiser (2017), en el cual se profundizará más adelante en este capítulo. 


\section{Supervisión en Colombia, estado actual}

En contraste con el prolífico desarrollo de literatura sobre supervisión en otras partes del mundo, en Latinoamérica, las publicaciones en torno a esta temática son pocas, y son principalmente de tipo teórico o documental (Bastidas-Bilbao y Velásquez, 2016; Bautista, 2014; Hernández, 2008; Loubat, 2005, en Chile; Rodríguez y Niño, 2006, en Colombia; Wielenska y Oshiro, 2012, en Brasil). Este nivel de producción en Latinoamérica puede estar relacionado con un bajo nivel de atención a los aspectos mencionados previamente en torno a la supervisión. Por esto, surge la necesidad de revisar la pertinencia de la formación para la supervisión clínica en Colombia, tomando como referencia las experiencias internacionales descritas.

Además, del poco desarrollo en la literatura, se han encontrado necesidades adicionales en la supervisión que se practica en Colombia. En un estudio realizado por Novoa-Gómez et al. (en preparación) se encuestaron a 64 supervisores de práctica clínica en psicología, en 25 programas de pregrado y posgrado, vinculados a universidades de diversas regiones de Colombia. El objetivo de este estudio fue analizar las prácticas en supervisión clínica en algunas categorías: objetivos, metodologías, elementos de la relación entre supervisor y supervisado, competencias del supervisado, evaluación, formación, conocimiento de los supervisados y discutir la pertinencia de la formación para la supervisión clínica de acuerdo con lo descrito por los modelos de entrenamiento basados en la evidencia. Los resultados de esta investigación muestran la necesidad de que los supervisores reciban entrenamiento formal en el desarrollo de competencias para la supervisión clínica. Estos hallazgos corroboran las afirmaciones de Reiser y Milne, "[los supervisores] muestran el desconocimiento de las mejores prácticas en el ejercicio de la supervisión, la escasa formación para ser supervisor y la necesidad de un programa de formación” (Reiser y Milne, 2017, p. 11). La necesidad de una supervisión clínica que garantice la protección de la sociedad y el desarrollo de competencias del terapeuta en formación, es acuciante. En primer lugar, porque, en Colombia, el Ministerio de Salud y Protección Social (2006), de acuerdo con la Ley 1090 de 2006 (Congreso de la República), autoriza a todos los psicólogos con el título de pregrado a ejercer toda actividad de enseñanza, aplicación e indicación del conocimiento psicológico y de sus técnicas específicas. Este hecho disminuye la posibilidad de garantizar las competencias clínicas que requieren los profesionales en este campo. En segundo lugar, ya que la formación de profesionales en áreas clínicas y de salud requieren cumplir de estándares de supervisión. 
En cuanto a la función de la supervisión, el estudio encontró que, frente al objetivo, ofrecer retroalimentación al supervisado, fue evaluado como el objetivo menos importante. Falender (2014b) resalta que el ofrecer retroalimentación positiva y negativa para el desarrollo de la supervisión es una de las competencias del supervisor que facilita la formación de las habilidades clínicas. En el estudio también se encontró que el objetivo de preservar el bienestar de los consultantes y el de la sociedad, no se distinguió de la formación de competencias en los supervisados. Mientras que el consenso internacional es que la protección del consultante es el rol primario de la supervisión (e. g., APA, 2015; Falender, 2014b). Estos dos aspectos, sumados a algunos que mencionaremos a continuación, señalan el desconocimiento de los supervisores de las mejores prácticas de supervisión.

En la categoría metodología, según Novoa-Gómez et al. (en preparación), frente a la forma de supervisión más usada, aparece la individual y la grupal y como la menos usada la virtual. Con respecto a las herramientas, la más usada es la indirecta y asincrónica (registros escritos de la sesión y reporte oral del supervisado), empleando menos la que se apoya en registros de video, audio, la observación en vivo y la coterapia, que son sugeridas como estrategias de supervisión por Borders et al. (2014) y Falender (2014b).

En la categoría formación recibida para la realización de la labor como supervisores clínicos y la percepción de la necesidad de ésta, según Novoa-Gómez et al. (en preparación), se constató una incoherencia en el $40 \%$ de los supervisores, al respecto. El 90,3\% manifiesta desconocer programas de formación o recursos y $50 \%$ afirma que cuenta con un tipo de formación para supervisar. Lo que parece poner en evidencia, que los supervisores no tienen presente que se requiere un entrenamiento específico para desarrollar las competencias para impartir la supervisión. Frente a la necesidad de formación, el 87,1\% manifiesta la necesidad de formación especializada y 69,4\% reconoce necesitar un entrenamiento en un modelo concreto de supervisión.

La ausencia de programas de formación y la necesidad de formación de los supervisores, sin embargo, no es muy diferente de lo que sucede en el resto del mundo. En el ámbito mundial, parece no haber programas que entrenen y evalúen el ejercicio de la supervisión al margen de un largo número de recomendaciones no sistemáticas, incluso cuando existe una investigación prolífera sobre el contenido a nivel metodológico y teórico de la supervisión (Falender y Shafranske, 2017; Milne et al. , 2011a). En dicho programa, el conocimiento, las habilidades y las actitudes de 
la supervisión deben ser sistemáticamente desarrollados y monitoreados a fin de asegurar el desarrollo de competencias de supervisión en el supervisor, el impacto positivo sobre la relación de supervisión y el mejoramiento en los resultados de la terapia y las competencias de supervisado (Falender y Shafranske, 2017).

La necesidad de programas de formación se hace evidente en diversas fuentes. Por ejemplo, en "EE. UU. se vio la importancia de investigar sobre la definición de las habilidades que debía tener un supervisor, la medición y el entrenamiento de éstas” (Fleming, Oliver y Bolton, 1996, p. 6). En Australia, Spence, Wilson, Cavanagh, Strong y Worrall (2001) hicieron un estudio con cuatro profesionales del área de salud mental, que les hizo ver la escasa información sobre las formas más eficaces para formar a los supervisores. En el seguimiento realizado por Milne y James (2002) a un supervisor experto en terapia cognitivo conductual, se encontraron falencias en su competencia, aunque contaba con acreditación de dos organizaciones profesionales. Falender y Shafranske (2004) afirman como resultado de un resumen de encuestas, que un entrenamiento formal en supervisión, no lo tienen la mayoría de los psicólogos en Estados Unidos. Fleming (citado en Milne, 2009a) anota que, en el Reino Unido, una encuesta arrojó que la mayoría de los supervisores contaba con un tipo de formación y entrenamiento, pero existía variación en el diseño de la formación recibida. En los datos anteriores, arrojados por estudios sobre la realidad de la supervisión en distintos países, se evidencia la necesidad de trabajar en la formación de los supervisores clínicos, expresada por Milne (2009a).

\section{Entrenamiento del modelo de supervisión basado en la evidencia (Milne y Reiser, 2017)}

En la educación y formación del psicólogo con un énfasis clínico en pregrado o preparación en el campo clínico en posgrado, es vital la práctica clínica supervisada, en la cual se inicia la atención de pacientes que consultan por diversas dificultades, con el acompañamiento de un profesional con experiencia en supervisión, que "busca que los servicios prestados al consultante sean de calidad y que se desarrollen las competencias adecuadas en el profesional en formación” (Falender y Shafranske, 2004, p. 3). Milne (2009b) enfatiza en que la supervisión clínica tiene un papel crítico en la formación del profesional y es altamente valorada, aunque se requiere mayor investigación y una mejora, ya que como lo señalaban Holloway y Poulin (citados en Milne, 2009b), esta ha sido un área de investigación descuidada, con muy pocos desarrollos y divulgación. 
Como se mencionó en la primera sección, uno de los programas de entrenamiento a supervisores que se destaca es el diseñado por Milne y Reiser (2017), que fue desarrollado basándose en estrategias de enseñanza aprendizaje similares a las sugeridas para la supervisión. Provee un modelo de supervisión que puede implementarse sistemáticamente y puede evaluarse de forma específica. A continuación, se describirá de forma muy general este modelo.

En primer lugar, se basa en el Modelo del Tándem, que en este contexto hace referencia a una bicicleta de dos puestos. De acuerdo con Milne y Reiser (2017), el supervisor tiene el puesto delantero, ya que debe ser quien guíe el proceso de aprendizaje. En el puesto trasero, va el supervisado, quien debe participar activamente del aprendizaje dentro de la supervisión. Además, las dos llantas del tándem involucran, respectivamente, los ciclos de la supervisión guiada por el supervisor y de aprendizaje llevado a cabo por el supervisado. La llanta delantera representa el ciclo de guía de la supervisión formativa que incluye los siguientes elementos: evaluación de necesidades, establecimiento de metas, empoderamiento del supervisado, facilitación del aprendizaje y monitoreo/evaluación del proceso. La llanta trasera representa el ciclo de aprendizaje del supervisado, que se basa en la clasificación del aprendizaje experiencial del Kolb (1984, citado en Milne, 2018): conceptualización, planeación, comportarse/experimentar, vivenciar y reflexionar.

En el entrenamiento desarrollado por Reiser y Milne, se emplearon seis principios que lo hacen ideal para su replicación. Estos son: a) Conceptualización cuidadosa: en la que se busca tener un concepto y un modelo claro de la supervisión que permita su diseminación; b) Operacionalización: lo que permite identificar los componentes de la supervisión y su medición; en este contexto, la operacionalización se hizo con la herramienta de medición supervisión: evaluación de la adherencia y la orientación (Supervision: Adherence and guidance evaluation, SAGE; Milne, Reiser, Cliffe y Raine, 2011); c) Utilizar la mejor evidencia disponible: se seleccionó información empírica que apunta a las mejores prácticas de formación, y se integraron con teorías apropiadas y el juicio de expertos para su organización; d) Interpretar la información ampliamente: para articular la información encontrada sobre supervisión clínica, se emplearon teorías

Modelo del Tándem, de acuerdo con Milne y Reiser (2017), el supervisor tiene el puesto delantero, ya que debe ser quien guíe el proceso de aprendizaje. En el puesto trasero, va el supervisado, quien debe participar activamente del aprendizaje dentro de la supervisióna. 
y conocimiento de áreas diferentes a la psicología con el fin de fortalecer y ampliar su impacto; e) Diseminar la información libremente: se han realizado esfuerzos por permitir el acceso al material de formación y divulgarlo en el campo internacional, y f) Garantizar la fidelidad: se han desarrollado manuales para incrementar la fidelidad del uso del SAGE y del modelo de supervisión basado en la evidencia.

El manual de entrenamiento de Milne y Reiser (2017), plantea seis temas generales que se pueden ofrecer en forma de un taller corto. Estos elementos son: a) establecimiento de una estructura para el entrenamiento, b) la alianza de trabajo en la supervisión, c) el establecimiento de objetivos, d) facilitar el aprendizaje, e) retroalimentación y evaluación y f) soporte y guía para el supervisado. Además, como recursos de apoyo brinda material para distribuir entre los participantes y videos de demostración de las habilidades mencionadas en el entrenamiento. A continuación, se presentará la aplicación de las recomendaciones de este manual en nuestra investigación.

\section{Aplicación de un programa de entrenamiento}

Este proyecto se enmarca en la línea de investigación sobre variables implicadas en procesos de formación en psicología, adscrita al grupo de investigación en Ciencias del Comportamiento de la Fundación Universitaria Konrad Lorenz. Se deriva de cuatro trabajos previos en los que se ha venido indagando sobre cuáles son las herramientas pedagógicas que contribuyen a la supervisión (competencias que debe tener el supervisor y el supervisado, los modelos de supervisión propuestos y aspectos de la relación supervisor-supervisado), cómo estas herramientas pedagógicas se emplean en la supervisión en Colombia, si se encuentra necesaria la formación para la supervisión clínica, y cómo podría ser esta formación para supervisores clínicos en nuestro contexto. En el primer trabajo, se realizó una revisión exploratoria (scoping review) de la literatura en supervisión clínica (Novoa-Gómez, Córdoba-Salgado, Peñuela et al., en preparación). En el segundo trabajo, se indaga sobre las prácticas de supervisores clínicos para la formación de competencias clínicas en programas de licenciatura y posgrado en Colombia (Novoa-Gómez et al., 2019). En el tercer trabajo, se analiza el tipo de competencias que se entrenan durante la supervisión, describiendo las clases de respuestas que ocurren entre el supervisor y el supervisado durante el proceso de supervisión para la formación de competencias del supervisado, basadas en los dominios de competencias propuestos por la APA (2006) (Novoa-Gómez, Córdoba-Salgado, Rojas et al., en revisión). Finalmente, en el cuarto trabajo, se describe la perspectiva de supervisores 
clínicos de diversas corrientes teóricas y terapéuticas en psicología, sobre las características de la supervisión, sus objetivos y competencias profesionales, a la luz de las condiciones del contexto actual de formación de psicólogos clínicos en pregrado y posgrado (Novoa-Gómez, Córdoba-Salgado, Robayo et al., en revisión). En el primer trabajo, Novoa-Gómez, Córdoba-Salgado, Peñuela et al. (en preparación) identificaron amplios y crecientes desarrollos en esta área de investigación en Estados Unidos, Canadá, Reino Unido, España y Australia, y desarrollos apenas incipientes en Latinoamérica, con menor desarrollo en Colombia, así como los modelos de supervisión que recogen las buenas prácticas y están basados en evidencia. En el segundo trabajo, Novoa-Gómez et al. (2019) manifiestan que los resultados muestran que la formación para la supervisión clínica es pertinente y necesaria en Colombia. El tercer trabajo permitió identificar de manera objetiva, la dedicación de tiempo a la formación de diversos tipos de competencia, requeridos para la práctica de psicología clínica basada en la evidencia, lo cual le permitirá a las instituciones universitarias y a los supervisores realizar acciones correctivas desde sus propios lineamientos (Novoa-Gómez, Córdoba-Salgado, Rojas et al., 2019). El cuarto trabajo permite afirmar que se requiere del acercamiento de los supervisores e instituciones a los programas de formación o actualización en supervisión pertinentes para la práctica en contexto. La importancia de esto radica en que la supervisión es la única experiencia clínica a la cual pueden acceder la mayoría de los terapeutas en formación; por tanto, al convertirlo en un proceso más eficiente, que genere un impacto favorecedor en todos los actores, contribuirá a la función social de la intervención psicológica. Con esta investigación, Quintero \& Novoa-Gómez (2018) aportan a la línea de investigación, en la medida que permita conocer en una muestra de supervisores clínicos que trabajan en una perspectiva cognitivo conductual, el efecto del taller desarrollado por Milne y Reiser (2017) en las competencias del supervisor en su labor docente. Los resultados permitirán también establecer si podría considerarse el taller, como un programa de entrenamiento que pueda seguirse replicando, sin la necesidad del componente de la supervisión a los supervisores, elemento propuesto por los autores, pero que sería de un costo económico significativo para el caso de las universidades en Colombia. De esta forma, se espera contribuir a la formación con calidad en las competencias que redunde en el aprendizaje de los profesionales en psicología clínica y en un cuidado del consultante.

\section{Participantes}

Participaron en esta investigación quince supervisores clínicos de pregrado que realizaban sus actividades de docencia en el centro de atención en psicología 
clínica adscrito a una institución universitaria en la ciudad de Bogotá. Se entiende como supervisor clínico a un profesional en psicología, con estudios de posgrado, que por lo menos ha tenido un año de trabajo o experiencia en el área clínica y está guiando el proceso de adquisición y evaluación de la competencia profesional de estudiantes de psicología clínica.

Los supervisores se asignaron en dos grupos de forma aleatoria, una vez se definieron los participantes que se encontraron disponibles e interesados en la investigación. Al grupo control se asignaron cinco supervisores clínicos universitarios de pregrado y teniendo en cuenta los supuestos de muerte experimental, al grupo experimental se asignaron diez supervisores clínicos universitarios de pregrado. Cada supervisor tuvo tres supervisados, para un total de 45 supervisados. Se excluyeron del estudio los supervisores de otro tipo de prácticas profesionales.

Flujo de los participantes

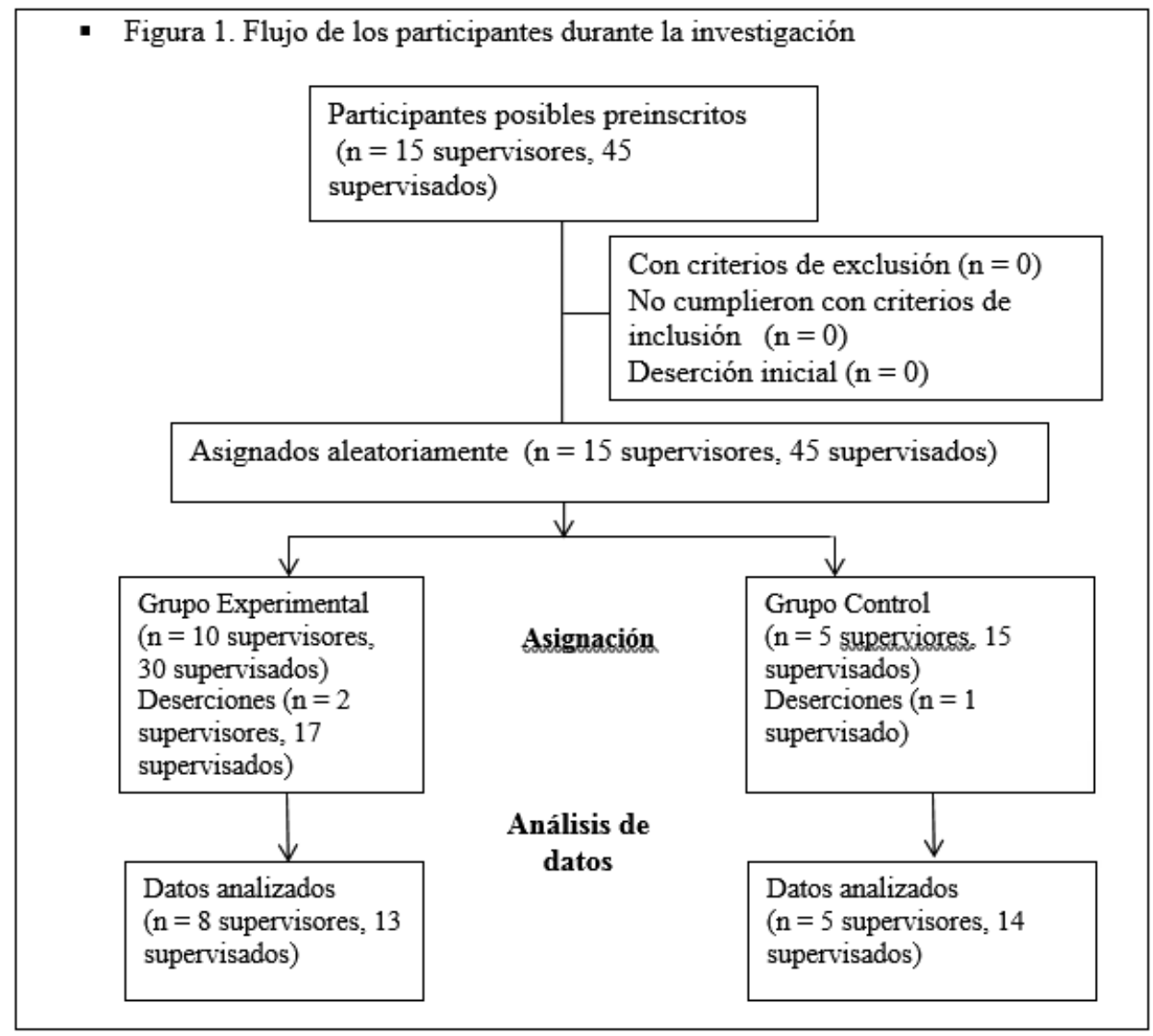

Figura 1. Flujo de los participantes durante la investigación 
Una vez realizadas las medidas pretest, la aplicación del taller y las medidas postest, como se observa en la Figura 1, quedaron 5 supervisores y 14 supervisados del grupo control (GC), 8 supervisores y 13 supervisados del grupo experimental (GE). Los supervisores descartados del GE fueron aquellos que no concluyeron el taller teórico-práctico en supervisión clínica cognitiva conductual del modelo de supervisión clínica basado en la evidencia (EBCS) (Milne y Reiser, 2017), y los supervisados descartados fueron aquellos que no diligenciaron los instrumentos o no pudieron ser grabados en una de las sesiones de la que se extrajo la medida.

\section{Instrumentos}

Supervisión: evaluación de la adherencia y la orientación (SAGE) heteroaplicada. El SAGE, según Milne y Reiser (2011), es un instrumento para medir la competencia en supervisión en relación con la terapia cognitivo-conductual y el enfoque de la supervisión clínica basada en la evidencia, que constó inicialmente de 23 ítems, ya que en las versiones posteriores se eliminó el ítem 9. El instrumento asume tres factores: los ítems 1 - 4 denominados factores comunes, miden la relación de supervisión o alianza; los ítems 5 - 18 denominados ciclo de supervisión, evalúan las competencias de supervisión, y los ítems 19 - 22 denominados ciclo de aprendizaje del supervisor, miden los signos iniciales del aprendizaje experiencial, ítems definidos dentro de un manual de codificación. Se debe calificar la competencia después de observar una sesión de supervisión, por medio de una cinta de audio o video y usando una escala tipo likert de 1 - 7 que va de incompetente a experto. También se pueden añadir notas que podrían explicar puntuaciones altas o bajas o sugerir maneras de mejorar lo observado en supervisión. Según Milne y Reiser (2017), el SAGE tiene una consistencia interna (basada en el alfa de Cronbach) en la escala completa de 0,92 y por subescalas: factores comunes 0,91 , ciclo de supervisión 0,91 y ciclo de aprendizaje 0,80.

SAGE autoaplicado. Con el fin de contar con una medida de autoevaluación del supervisor antes y después de la intervención, para esta investigación se toman del SAGE los ítems 1 al 18, que valoran las competencias de los factores comunes que miden la relación de supervisión o alianza y el ciclo de supervisión que evalúan las competencias de supervisión. Cada supervisor autocalifica una a una las competencias, usando una escala tipo likert de 1 - 7 que va de incompetente a experto. 
Encuesta de impacto de la supervisión (SOS). La encuesta de impacto de la supervisión SOS, según Falender y Shafranske (2004), evalúa la opinión de los supervisados sobre la supervisión, consta de 20 ítems y usa una escala tipo likert de 1 a 7. Se sugiere, por parte de sus autores, usarla en intervalos durante la supervisión o como una medida permanente. Reese et al. (2009) evaluaron la consistencia del SOS con coeficiente alfa en 0,92 y 0,98 .

\section{Variables del estudio}

\section{Variable independiente}

La variable independiente para esta investigación fue la participación en el taller teórico-práctico en supervisión clínica cognitiva conductual del modelo de supervisión clínica basado en la evidencia (EBCS) (Milne y Reiser, 2017). El EBCS cuenta con un manual que concreta cómo los supervisores pueden ser entrenados en el enfoque, por medio de un taller de seis módulos que contienen sus respectivas presentaciones de PowerPoint, material de lectura y grabaciones de video de los aspectos claves de la supervisión.

\section{Variable dependiente}

La variable dependiente para este estudio es la percepción del supervisado respecto a la calidad de la supervisión, medida mediante el SOS. Así como las competencias evaluadas por el SAGE en su forma aplicada y heteroaplicada. Las competencias del SAGE se presentan a continuación:

Relación: el supervisor asegura que las “condiciones básicas” (es decir, calidez, autenticidad, empatía y comprensión) estén presentes. Asimismo, asegura que cualquier emoción que surja en la supervisión o en la terapia o trabajo del supervisor sea reconocida y manejada adecuadamente.

Colaboración: el supervisor anima al supervisado a ser un socio activo en la experiencia de la supervisión (es decir, tener tareas y objetivos compartidos). Hay pruebas claras de trabajo en equipo productivo, con el supervisor animando al supervisado a participar plenamente. Esto asegura que el supervisado tome el nivel apropiado de responsabilidad y control, con respecto a su experiencia de aprendizaje.

Gestión: el supervisor se involucra en la actividad de estructuración y estimulación que establece el orden en la sesión de supervisión (por ej., introducción de 
un tema o creación de una tarea, actividad estructural o señalando afirmaciones) y establece situaciones de aprendizaje (por ej., creación u organización de materiales didácticos). El supervisor al igual se asegura de que el ritmo de sesión sea apropiado y que las sesiones fluyan de manera adecuada (función "normativa”).

Facilitación: el supervisor fomenta las reacciones y emociones deseables, como la curiosidad o la motivación para aprender, para facilitar el aprendizaje (por ej., a través de la profundización de las reflexiones del supervisor: función “formativa”).

Orden de la agenda: el supervisor establece las metas de aprendizaje explícitas para la sesión de manera colaborativa y administra la agenda de la sesión revisando las necesidades del supervisado (basándose en las sesiones anteriores). El supervisor se asegura de que los temas se acuerden de una manera apropiada, se definan y se aborden adecuadamente.

Demostración: el supervisor desarrolla la competencia demostrando la ejecución correcta de una habilidad (por ej., con material ilustrativo de audio/video o modelando: modo icónico). Estas actividades también pueden ayudar al supervisado a identificar obstáculos potenciales y reflexionar sobre los mecanismos de cambio que sustentan las tareas que él/ella está usando con los pacientes.

Discusión: el supervisor discute los problemas para desestabilizar y transformar de manera hábil y constructiva el conocimiento del supervisado. Típicamente, ocurre como una reacción a la opinión del supervisado, a la práctica clínica o a la comprensión de los hechos. Discutir a menudo implica el uso de cuestionamiento socrático, diseñado para ser abierto y para aumentar la conciencia del supervisado de cuestiones claves o preocupaciones (sin imponer una opinión o ejercer la autoridad).

Evaluación: el supervisor monitorea, comprueba, evalúa explícitamente el trabajo o la competencia del supervisado.

Retroalimentación (dar): el supervisor explica el propósito de la retroalimentación, realiza una corrección precisa, una sugerencia o demostración.

Retroalimentación (recibir): el supervisor le pide al supervisado que resuma sus percepciones de la sesión de supervisión. La forma en que se obtiene esta información debe ser abierta, lo que anima al supervisado a ser honesto y próximo acerca de sus opiniones e impresiones, tanto de la supervisión como del proceso de 
aprendizaje en general. El supervisor provoca activamente comentarios no solo sobre aspectos útiles de la sesión de supervisión, sino también sobre cualquier dificultad o conflicto que se haya experimentado.

Formulación: el supervisor trabaja activamente para ayudar al supervisado a desarrollar una formulación de cada caso. Los supervisores pueden ayudar a los supervisados a desarrollar la formulación de casos mediante una serie de enfoques. Como resultado, el supervisado debe ser capaz de definir y explorar los problemas y darles sentido.

Escuchar: el supervisor escucha activamente y presta mucha atención al habla y comportamiento del supervisado. El supervisor escucha de una forma segura y auténtica antes de reaccionar. El supervisor está enfocado y no distraído tratando de ser "multi-tarea”, es decir, realizando otras actividades. La escucha efectiva está indicada por elementos como el resumen, parafraseo y cuestionamiento por parte del supervisor.

Observación: el supervisor observa la actividad del supervisado en sesiones de terapia, ya sea en vivo o por medio de grabaciones de video o audio.

Sugerencias: el supervisor le recuerda o guía al supervisado sobre el material relevante que necesite sugerirle.

Cuestionamiento: el supervisor ayuda al supervisado a desarrollar hipótesis sobre problemas terapéuticos y a generar posibles soluciones. El supervisado es asistido en el desarrollo de un conjunto de perspectivas con respecto al proceso terapéutico y la utilidad de diferentes técnicas terapéuticas. Una mayor conciencia de sí mismo o un mayor compromiso crítico (con los conceptos o métodos que se utilizan) también puede resultar de un cuestionamiento efectivo

Enseñanza: el supervisor proporciona información al supervisado de manera didáctica ("transmisión de información”), que puede ser sobre teorías, hechos, ideas, métodos o artículos de investigación. Incluye el uso de grabaciones de video o audio (por ej., explicando una idea).

Entrenamiento: el supervisor ayuda al supervisado a aprender involucrándolo en una actividad experiencial apropiada. El método debe apropiarse para las necesidades de aprendizaje del supervisado y su etapa de desarrollo, y por tanto debe 
basarse en las fortalezas. El supervisor se involucra en los métodos de "acción” pertinentes, incluyendo: simulaciones, ensayos conductuales y juegos de roles educativos.

Vivenciar: el supervisor ayuda al supervisado a comprender sus experiencias en la supervisión y en relación con lo sugerido en la sesión con el paciente.

\section{Diseño y tipo de estudio}

El diseño que se utilizó en el presente trabajo de investigación fue experimental de grupo control en lista de espera, con pretest-postest:

$\begin{array}{cccc}\text { GE } & \text { 01 } & \mathrm{X} & \mathrm{O2} \\ \text { GC } & 03 & - & 04\end{array}$

Este diseño tiene dos grupos: el grupo experimental recibe el tratamiento y el grupo control en lista de espera no lo recibe. Cada grupo realizó dos autoevaluaciones, prestest y postest, por parte del supervisor con el SAGE autoaplicado; dos evaluaciones, prestest y postest, a partir de la observación y calificación de las grabaciones de dos sesiones de supervisión; finalmente, dos evaluaciones, prestest y postest, de cada supervisado hacia su supervisor con el instrumento SOS. El efecto de la intervención se refleja en el cambio en las medidas del prestest al postest en el grupo control y en el postest entre el grupo control y experimental (Kazdin, 2001). La asignación de los participantes a los grupos del experimento se realizó al azar.

\section{Análisis de resultados}

El análisis de datos se hizo por medio del programa estadístico SSPS 23.0. Se utilizó la prueba no paramétrica U de Mann Whitney para dos muestras independientes y la prueba no paramétrica de Friedman para muestras relacionadas. Las muestras independientes fueron las comparaciones de las medias de los puntajes arrojados por los instrumentos SAGE autoaplicado, SAGE heteroaplicado y SOS, entre los grupos control y experimental, en el prestest y postest. Las muestras relacionadas fueron las comparaciones de las medias de los puntajes arrojados por los instrumentos SAGE autoaplicado, SAGE heteroaplicado y SOS, entre el prestest y postest del grupo control y del experimental. 


\section{Procedimiento}

La investigación se desarrolló en tres fases:

Fase 1: se identificó dentro de los lineamientos para las mejores prácticas de supervisión, un modelo de supervisión clínica que tuviera como fin desarrollar las competencias, mejorar el desarrollo de la práctica clínica y que tuviera un programa de entrenamiento a supervisores con una historia de intervención con resultados evidentes. Identificado el modelo de supervisión clínica, con su respectivo programa de entrenamiento, se procedió a realizar las traducciones del material de entrenamiento (por el equipo de investigación perteneciente a la línea de investigación y con la asesoría de los docentes que dirigen la investigación) y de los respectivos instrumentos de medición (para el caso de los instrumentos, primero se hizo el procedimiento de traducción y retrotraducción y luego se entregaron a las personas seleccionadas para su evaluación y concepto, en lo que concierne al proceso de validación de contenido con juicio de expertos). Posteriormente, se prepararon los materiales para la aplicación del taller. Se construyó la base de datos para análisis de resultados, con el fin de ir alimentándola con la aplicación del prestest.

Fase 2: tras la convocatoria abierta a los supervisores clínicos universitarios que desarrollaban su labor docente en el centro de psicología clínica de una institución de educación superior en la ciudad de Bogotá, se realizó durante dos horas la introducción al taller de forma presencial (se presentaron los resultados de las investigaciones anteriores que hacían ver la importancia de la formación de los supervisores clínicos, al igual que el sustento teórico del modelo y la dinámica del taller, se asignaron al azar 15 supervisores clínicos a los grupos control lista de espera -5 supervisores - y experimental -10 supervisores - , cada supervisor con 3 supervisados, se realizó el diligenciamiento del consentimiento informado y se tomó una de las medidas del prestest con el instrumento SAGE adaptado a la lista de chequeo).

Mediante el correo se recogió la información sobre los horarios de supervisión y los posibles horarios de los que disponían los supervisores para el taller presencial; de igual forma se contacta telefónicamente a los docentes que por medio del correo fue difícil obtener la información. Se asistió a la primera supervisión grupal de introducción, con el fin de explicar a los supervisados de cada supervisor el fin del estudio, el proceso a desarrollar, solucionar dudas presentadas y diligenciar los consentimientos informados como supervisados. 
Posteriormente, se asistió a una sesión de supervisión con el fin de grabarla y de aplicar a los supervisados la SOS, como otras medidas de prestest. La primera aplicación de la SOS a los supervisados y la grabación de la sesión de supervisión coinciden con las primeras sesiones de supervisión.

Dado que no fue posible establecer un consenso para la aplicación del taller a los supervisores, se adapta el taller para su aplicación de manera virtual. Con el grupo experimental, se pone a disposición los seis módulos a través de un aula virtual, para que cada supervisor los fuera desarrollando de manera gradual durante tres semanas. Los módulos contenían la información sobre las distintas competencias que debe desarrollar un supervisor de acuerdo con el modelo, así como material audiovisual que muestra la ejecución de las competencias y ejercicios que favorecían el desarrollo de las mismas. Los docentes que dirigían la investigación y que tienen experiencia en supervisión y conocen el modelo y los módulos, estaban disponibles a solucionar las dudas e inquietudes que se presentaran. El equipo de investigación revisaba el cumplimiento de las actividades y a través de contacto telefónico o correo se indagaba por los incumplimientos. Se tuvo dificultad con algunos supervisores que por distintos motivos no desarrollaron los módulos del taller dentro de los tiempos establecidos, por lo cual se debió dar dos semanas más, para que los supervisores pudieran completar el taller.

Terminada la aplicación del taller, se tomaron las medidas del postest con el instrumento SAGE autoaplicado para los supervisores, la SOS a los supervisados de cada supervisor y se hizo la grabación en video de una sesión de supervisión. Esta aplicación tuvo lugar durante las dos últimas semanas del semestre académico.

Se evaluaron las grabaciones de supervisión en video, empleando el SAGE heteroaplicado. Para ello, cinco personas, entre los que se contaban los docentes a cargo de la línea de investigación, los auxiliares de investigación y un estudiante de maestría, observaron varios videos y los calificaron independientemente. Luego, se compararon los puntajes en cada uno de los ítems y se discutieron los desacuerdos a la luz de las indicaciones dadas en el manual del SAGE. Una vez se alcanzó un acuerdo entre observadores apropiado, se calificaron los videos en parejas, con el fin de obtener una medida de acuerdo entre observadores en todos los videos.

Fase 3: cuando se obtuvieron los datos recolectados en su totalidad, se procedió primero a descartar del GE los supervisores que no concluyeron el taller teórico-práctico en supervisión clínica cognitiva conductual del modelo de supervisión 
clínica basado en la evidencia (EBCS) (Milne y Reiser, 2017) y a descartar los supervisados del GC y GE que no diligenciaron los instrumentos o no pudieron ser grabados en la sesión de supervisión. Como segundo paso se analizaron los resultados.

\section{Resultados}

Para contribuir a la evidencia al respecto, esta investigación buscaba establecer el efecto del taller teórico-práctico en supervisión clínica cognitiva conductual del modelo de supervisión clínica basado en la evidencia (EBCS) (Milne y Reiser, 2017), en las competencias desplegadas por el supervisor clínico en la interacción con el supervisado.

Para ello se hizo el análisis con la prueba no paramétrica U de Mann Whitney para dos muestras independientes y la prueba no paramétrica de Friedman para muestras relacionadas. Los resultados muestran que hubo equivalencia entre el grupo experimental y el grupo control en las medidas empleadas. Sin embargo, tampoco se encontraron diferencias significativas al comparar las medidas de los diferentes componentes del SAGE autoaplicado y heteroaplicado y del SOS, tanto entre prestest y el postest en el GE y las comparaciones postest entre el grupo control y el grupo experimental (véanse Tablas 1 y 2).

Tabla 1. Significancia estadística de las comparaciones de las medidas de lápiz y papel

\begin{tabular}{l|c|c|c|c|c|c|c|c}
\multirow{2}{*}{} & \multicolumn{4}{|c|}{ GC frente a GE } & \multicolumn{4}{c}{ Pretest frente a Postest } \\
\cline { 2 - 9 } & \multicolumn{2}{|c|}{ Pretest } & \multicolumn{2}{c|}{ Postest } & \multicolumn{3}{c}{ GC } & \multicolumn{2}{c}{ GE } \\
\hline & $\mathrm{U}$ & $\mathrm{p}$ & $\mathrm{U}$ & $\mathrm{p}$ & $\chi^{2}$ & $\mathrm{p}$ & $\chi^{2}$ & $\mathrm{p}$ \\
\hline SAGE ES & 13,5 & 0,158 & 20,0 & 0,583 & 1,80 & 0,18 & 0,40 & 0,527 \\
\hline SAGE PS & 15,5 & 0,244 & 22,5 & 0,759 & 1,80 & 0,18 & 0,40 & 0,527 \\
\hline SOS & 182,5 & 0,393 & 136,5 & 0,114 & 0,33 & 0,564 & 0,04 & 0,827
\end{tabular}

Nota: GE: grupo experimental; GC: grupo control; ES: estructura de la supervisión; PC: proceso de la supervisión. 
Tabla 2. Significancia estadística de las comparaciones de la medida de observación SAGE heteroaplicado

\begin{tabular}{l|c|c|c|c|c|c|c|c} 
& \multicolumn{3}{c|}{ GC frente a GE } & \multicolumn{4}{c}{ Pretest frente a Postest } \\
\cline { 2 - 10 } & \multicolumn{2}{|c|}{ Pretest } & \multicolumn{2}{c|}{ Postest } & \multicolumn{3}{c}{ GC } & GE \\
& $\mathrm{U}$ & $\mathrm{p}$ & $\mathrm{U}$ & $\mathrm{p}$ & $\chi^{2}$ & $\mathrm{p}$ & $\chi^{2}$ & $\mathrm{p}$ \\
\hline SAGE ES & 564,5 & 0,269 & 405 & 0,52 & 0,360 & 0,549 & 0,571 & 0,45 \\
\hline SAGE PS & 449 & 0,607 & 344 & 0,123 & 0,926 & 0,336 & 0,034 & 0,853 \\
\hline SAGE CA & 511 & 0,583 & 335,5 & 0,194 & 0,154 & 0,695 & 0,143 & 0,705 \\
\hline
\end{tabular}

Nota: GE: grupo experimental; GC: grupo control; ES: estructura de la supervisión; PC: proceso de la supervisión; CA: ciclo de aprendizaje.

\section{Conclusiones}

La supervisión clínica brindada por un profesional con experiencia "busca que los servicios prestados al consultante sean de calidad y en el futuro profesional o profesional en formación se desarrollen las competencias adecuadas" (Falender y Shafranske, 2004, p. 3); por tanto, es relevante la necesidad de formar a los supervisores y realizar un trabajo formal sobre este tema. Una de las propuestas de formación desarrolladas a escala internacional y que cuenta con todos los elementos necesarios para una formación, es el taller teórico-práctico en supervisión clínica cognitivo conductual basado en la evidencia. Este es útil para promover o potenciar las competencias para el ejercicio de la supervisión y evidenciar el efecto de las competencias trabajadas en la formación del supervisor en la interacción con el supervisado mediante las sesiones de supervisión.

Frente a estudios que den cuenta de la aplicación de la propuesta de formación para supervisores, Milne (2010) comenta de un estudio piloto que realizó aplicando el taller teórico-práctico en supervisión clínica cognitiva conductual del modelo de supervisión clínica basado en la evidencia, con 256 supervisores, abordando los problemas en la formación como supervisores clínicos. Asimismo, menciona que los instructores dieron al manual una calificación de aceptabilidad del 74\% y los supervisores dieron al taller una calificación del 77\% de satisfacción. Milne (2010) identifica que las reacciones pueden decir poco sobre el aprendizaje de los participantes, sobre la transmisión de lo aprendido a las rutinas de supervisión o como se relaciona con la práctica terapéutica y su eficacia, aunque contribuye a la escasa 
literatura sobre capacitación de supervisores, desarrollo y difusión de un manual dentro de un enfoque aceptable para la supervisión y sobre el entrenamiento de supervisores. Por esto, el autor sugiere continuar con estudios que incluyan el impacto del taller sobre el aprendizaje y la transferencia a la práctica de supervisión.

En la presente investigación, sin embargo, los resultados muestran que la aplicación del taller no generó diferencias en las estrategias de los supervisores. Estos resultados pueden estar relacionados con el cambio en la modalidad del taller. Puesto que el uso de la modalidad virtual modificó algunas variables de aplicación que se tenían previstas inicialmente. Por ejemplo, durante la aplicación se observó que algunos supervisores realizaron las diferentes actividades de aprendizaje en el lapso de unos pocos días, lo que pudo dificultar la aprehensión de los conocimientos que se esperaban compartir. Por otro lado, el taller presencial presenta la oportunidad de practicar la habilidad que se ha enseñado, recibir retroalimentación y discutirla con los pares. Esta posibilidad se vio limitada de manera importante por la modalidad. Si bien se habilitaron foros de discusión respecto a las actividades de aprendizaje en el aula virtual, se observó que la interacción entre los participantes en los foros fue escasa.

Por otra parte, la medida postest ocurrió en las dos últimas semanas del semestre, lo cual coincidió con las sesiones de supervisión que están destinadas a aspectos administrativos como revisión de historias clínicas, retroalimentación general del desempeño y entrega de notas. Este aspecto no permitió que los supervisores desplegaran las estrategias y habilidades que pretendieron formar a través del taller.

Las variables relevantes para la supervisión es el apoyo al supervisor por parte de las instituciones, ya que este es un factor mediador del impacto de la supervisión.
Dentro del modelo de supervisión basado en la evidencia (Milne, 2018), se observa que una de las variables relevantes para la supervisión es el apoyo al supervisor por parte de las instituciones, ya que este es un factor mediador del impacto de la supervisión. En este contexto, se observa un proceso paralelo. Si bien, los investigadores contaron con recursos valiosos y fundamentales para la aplicación del taller, tales como tiempo financiado de investigación, los recursos virtuales y auxiliares de investigación, los participantes no contaron con tiempo para participar del taller. Como se mencionó, esto pudo reducir el impacto del taller. 
En este sentido, se sugiere continuar con las investigaciones, de forma que se aplique el taller de manera presencial. Milne y Reiser (2017) indican que se deben incluir componentes teóricos y experienciales que promuevan el aprendizaje y se debe tener en cuenta como mínimo cinco puntos: primero, la definición de objetivos y metas claras al inicio de la formación; segundo, instalaciones, materiales de formación y liderazgo efectivo; tercero, utilizar métodos de entrenamiento que facilite el aprendizaje experiencial; cuarto, ofrecer retroalimentación de manera que los participantes al taller puedan comprender las competencias, y quinto, evaluar la eficacia del taller con el fin de valorar el logro de metas e identificar mejoras.

Al igual, se sugiere grabar durante el periodo académico todas las sesiones de supervisión (no solo dos), para ser calificadas con el SAGE, lo que permitiría evidenciar en un continuo de supervisiones las competencias del supervisor desplegadas en la interacción con el supervisado. Esto debido a que es difícil que, en una supervisión, el supervisor exhiba todas las competencias entrenadas en el taller y que se miden con los instrumentos mencionados.

Asimismo, se sugiere un tercer grupo experimental que tenga un acompañamiento al supervisor (supervisión al supervisor) después de la formación por parte de un guía que pueda retroalimentar el resultado de las mediciones, buscando mejorar las competencias en el ejercicio profesional y realizar la comparación no solo con el grupo control en lista de espera, sino también con este tercer grupo. Esto permitirá analizar si con el taller presencial, se pueden obtener resultados semejantes al grupo que contaría con supervisión al supervisor, lo cual podría abrir un camino para ofrecer el taller sin supervisión posterior, que puede hacer el proceso largo y costoso.

\section{Referencias}

American Psychological Association (APA). (2015). Guidelines for clinical supervision in health service psychology. The American Psychologist, 70, 33-46. doi: 10.1037/ a0038112.

Ballesteros, B. P., Muñoz-Martínez, A. M., Novoa-Gómez, M. M., Bazzani-Orrego, D., Brandwayn-Briceño, N. E., Lasso-Báez, A., Pachón-Basallo, M., \& Restrepo-Vélez, D. (2015). Características de la supervisión en la psicología clínica conductual (artículo inédito). En evaluación revista Psiké. 
Bastidas-Bilbao, H., \& Velásquez, A. M. (2016). Modelo lógico de la supervisión como actividad educativa para la formación en psicología clínica: conceptions of clinical supervisors. Avances en Psicología Latinoamericana, 34(2), 293-314. http://dx.doi.org/10.12804/apl34.2.2016.06.

Bautista, A. M. (2014). Movilización de los aprendizajes de terapeutas en formación que surgen de las conversaciones reflexivas a partir de la emergencia del observador en el contexto de supervisión. Quaestiones Disputatae: temas en debate, 7(14), 67-80.

Bernard, J. M., \& Goodyear, R. J. (2014). Fundamentals of clinical supervision (5 ${ }^{\text {th }}$ ed.). Boston, MA: Pearson.

Borders, L. D., Glosoff, H. L., Welfare, L. E., Hays, D. G., DeKruyf, L., Fernando, D. M., \& Page, B. (2014). Best practices in clinical supervision: Evolution of a counseling specialty. The Clinical Supervisor, 33(1), 26-44. http://dx.doi.org/10.10 80/07325223.2014.905225.

Boswell, J. F., Nelson, D. L., Nordberg, S. S., McAleavey, A. A., \& Castonguay, L. G. (2010). Competency in integrative psychotherapy: Perspectives on training and supervision. Psychotherapy: Theory, Research, Practice, Training, 47(1), 3. http://dx.doi.org/10.1037/a0018848.

Callaghan, G. M. (2006a). The functional idiographic assessment template (FIAT) system: For use with interpersonally-based interventions including functional analytic psychotherapy (FAP) and FAP-enhanced treatments. The Behavior Analyst Today, 7(3), 357. http://dx.doi.org/10.1037/h0100160.

Callaghan, G. (2006b). Functional analytic psychotherapy and supervision. International Journal of Behavioral and Consultation Therapy, 3(2), 416-431. http:// dx.doi.org/10.1037/h0100794.

Callahan, J. L., Neumann, C. S., Cox, R. J., \& Ruggero, C. J. (2017). The supervisor trainee quarterly evaluation (STQE): Psychometric support for use as a measure of competency. Training and Education in professional Psychology. http://dx. doi.org/10.1037/tep0000140.

Carmel, A., Villatte, J. L., Rosenthal, M. Z., Chalker, S., \& Comtois, K. A. (2016). Applying technological approaches to clinical supervision in dialectical behavior therapy: A randomized feasibility trial of the bug-in-the-eye (BITE) model. Cognitive and Behavioral Practice, 23(2), 221-229. http://dx.doi.org/10.1016/j. cbpra.2015.08.001. 
Colegio Colombiano de Psicólogos, C. C. (2006). Ley 1090 de 2006. Recuperado el 19 de febrero de 2017.

Ellis, M. V., Berger, L., Hanus, A., Alaya, E. E., Siembor, M. J., \& Swords, B. A. (2014). Inadequate and harmful clinical supervision: Revising the framework and assessing occurrence. The Counseling Psychologist, 42, 434-472. http://dx.doi. org/10.1177/0011000013508656.

Falender, C. A., Cornish, J. A. E., Goodyear, R., Hatcher, R., Kaslow, N. J., Leventhal, G. Shafranske, E., Sigmon, S., Stoltenberg, C., \& Grus, C. (2004). Defining competencies in psychology supervision: A consensus statement. Journal of Clinical Psychology, 60(7), 771-785. doi: 10.1002/jclp.20013.

Falender, C. A. (2014a). Supervision outcomes: Beginning the journey beyond the emperor's new clothes. Training and Education in Professional Psychology, 8(3), 143-148. http://dx.doi.org/10.1037/tep0000066.

Falender, C. A. (2014b). Clinical supervision in a competency-based era. South African Journal of Psychology, 44(1), 6-17. doi: 10.1177/0081246313516260.

Falender, C. A., \& Shafranske, E. P. (2004). Clinical supervision a competency - based approach. Washington, United Estates of America: American Psychological Association.

Falender, C. A., \& Shafranske, E. P. (2014). Clinical supervision in the era of competence. In W. B. Johnson \& N. Kaslow (Eds.), Oxford Handbook of Education and Training in Professional Psychology. Oxford. http://dx.doi.org/10.1093/ oxfordhb/9780199874019.013.022.

Falender, C. A., \& Shafranske, E. P. (2017). Competency-based clinical supervision: Status, opportunities, tensions, and the future. Australian Psychologist, 52(2), 86-93. doi: 10.1111/ap.12265.

Falke, S. I., Lawson, L., Pandit, M. L., \& Patrick, E. A. (2015). Participant supervision: Supervisor and supervisee experiences of cotherapy. Journal of Marital and Family Therapy, 41(2), 150-162. doi: 10.1111/jmft.12039.

Fleming, R. K., Oliver, J. R., \& Bolton, D. M. (1996). Training supervisors to train staff: A case study in a human service organization. Journal of Organizational Behaviour Management, 16, 3-25. 
Genuchi, M. C., Rings, J. A., Germek, M. D., \& Cornish, J. A. E. (2015). Clinical supervisors' perceptions of the clarity and comprehensiveness of the supervision competencies framework. Training and Education in Professional Psychology, 9(1), 68-76. http://dx.doi.org/10.1037/tep0000064.

Goodyear, R. K., \& Rodolfa, E. (2012). Negotiating the complex ethical terrain of clinical supervision. In S. J. Knapp, M. C. Gottlieb, M. M. Handelsman \& L. D. VandeCreek (Eds.), APA handbooks in psychology. APA handbook of ethics in psychology, vol. 2. Practice, teaching, and research (pp. 261-275). http://dx.doi .org/10.1037/13272-013.

Hernández, P. (2008). The cultural context model in clinical supervision. Training and Education in Professional Psychology, 2(1), 10-17. http://dx.doi. org/10.1037/1931-3918.2.1.10.

Holt, H., Beutler, L. E., Kimpara, S., Macías, S., Haug, N. A., Shiloff, N., \& Stein, M. (2015). Evidence-based supervision: Tracking outcome and teaching principles of change in clinical supervision to bring science to integrative practice. Psychotherapy, 52(2), 185-189. http://dx.doi.org/10.1037/a0038732.

Kohlenberg, R. J., \& Tsai, M. (1991). Functional analytic psychotherapy: A guide for creating intense and curative therapeutic relationships. New York: Plenum.

Kohrt, B. A., Jordans, M. J., Rai, S., Shrestha, P., Luitel, N. P., Ramaiya, M. K., ..., \& Patel, V. (2015). Therapist competence in global mental health: Development of the enhancing assessment of common therapeutic factors (ENACT) rating scale. Behaviour Research and Therapy, 69, 11-21. http://dx.doi.org/10.1016/j. brat.2015.03.009.

Loubat, T. (2005). Supervisión en psicoterapia: una posición sustentada en la experiencia clínica. Terapia Psicológica, 23(2), 75-84.

Martino, S., París, M., Áñez, L., Nich, C., Canning-Ball, M., Hunkele, K., \& Carroll, K. M. (2016). The effectiveness and cost of clinical supervision for motivational interviewing: A randomized controlled trial. Journal of Substance Abuse Treatment, 68, 11-23. http://dx.doi.org/10.1016/j.jsat.2016.04.005.

Milne, D. L. (2009a). Can we enhance the training of clinical supervisors? A national pilot study of an evidence-based approach. Clinical Psychology and Psychotherapy, 17, 321-328. doi: 10.1002/cpp.657. 
Milne, D. L. (2009b). Evidence - based clinical supervision: Principles and practice. Malaysia: The British Psychological Society and Blackwell Publishing Ltd.

Milne, D. L. (2010). Can we enhance the training of clinical supervisors? A national pilot study of an evidence - based approach. Clinical Psychology and Psychotherapy, $17,321-328$.

Milne, D. (2018). Evidence-based clinical supervision: Principles and practice. Leicester, England: Malden Blackwell Publishing.

Milne, D. L., \& James, I. A. (2002). The observed impact of training on competence in clinical supervision. British Journal of Clinical Psychology, 41, 55-72. http:// dx.doi.org/10.1348/014466502163796.

Milne, D. L., \& Reiser, R. (2017). A manual for evidence - based CBT supervision. United Estates of America: WILEY - Blackwell.

Milne, D. L., Reiser, R. P., Cliffe, T., \& Raine, R. (2011). SAGE: Preliminary evaluation of an instrument for observing competence in CBT supervision. The Cognitive Behavior Therapist, 4, 123-138.

Milne, D. L., Sheikh, A. I., Pattison, S., \& Wilkinson, A. (2011). Evidence-based training for clinical supervisors: A systematic review of 11 controlled studies. The Clinical Supervisor, 30(1), 53-71. http://dx.doi.org/10.1080/07325223.2011.564955.

Ministerio de Salud. (2015). Encuesta Nacional de Salud Mental. http://www.visiondiweb.com/insight/lecturas/Encuesta_Nacional_de_Salud_Mental_Tomo_I.pdf.

Ministerio de Salud y Protección Social. (2006). Ley 1090 de 2006. https://www.colpsic. org.co/aym.../LEY_1090_DE_2006_actualizada_junio_2015.pdf.

Myers, J. E., \& Sweeney, T. J. (2008). Wellness counseling: The evidence base for practice. Journal of Counseling \& Development, 86(4), 482-493. doi: 10.1002/j.15566678.2008.tb00536.x.

Newman, C. F. (2010). Competency in conducting cognitive-behavioral therapy: Foundational, functional, and supervisory aspects. Psychotherapy: Theory, Research, Practice, Training, 47(1), 12. http://dx.doi.org/10.1037/a0018849.

Newman, C. F. (2013). Training cognitive behavioral therapy supervisors: Didactics, simulated practice, and "meta-supervision". Journal of Cognitive Psychotherapy, 27(1), 5-18. https://doi.org/10.1891/0889-8391.27.1.5. 
Novoa-Gómez, M., Córdoba-Salgado, O., Rojas, N., Sosa, L., Cifuentes \& D. A., Robayo, S. (2019). A descriptive analysis of the interaction in clinical supervision. Frontiers in Psychology, 10:669 . https://doi: 10.3389/fpsyg.2019.00669

Novoa-Gómez, M., Córdoba-Salgado, O., Robayo, S. Rojas, N.; Mendoza, C. Supervisión clínica: La perspectiva del supervisor en psicología clínica. (2019) Suma Psicologica. (en revisión)

Novoa-Gómez, M., Córdoba-Salgado, O., Rojas, N., Sosa, L., Cifuentes, D. A., Robayo, S., \& Pardo, N. (en preparación). Competencias profesionales en psicología clínica: pertinencia de la formación para la supervisión clínica. Fundación Universitaria Konrad Lorenz. En revisión.

Novoa-Gómez, M., Córdoba-Salgado, O., Peñuela, C., González, M. E., Rodríguez, J., Rojas, N., Sosa, L., \& Cifuentes, D. (en revisión). Revisión sistemática de la producción científica en supervisión clínica (1982-2017).

Plakun, E. M., Sudak, D. M., \& Goldberg, D. (2009). The Y model: An integrated, evidence-based approach to teaching psychotherapy competencies. Journal of Psychiatric Practice ${ }^{\circledR}, 15(1)$, 5-11. doi: 10.1097/01.pra.0000344914.54082.eb.

Quintero, M. \& Novoa-Gómez, M (2018). Efecto de un programa de entrenamiento en supervisión clínica basado en la evidencia (Milne \& Reiser, 2017) en las competencias desplegadas por el supervisor clínico en la interacción con el supervisado. Tesis de Maestria en psicología clínica. Fundación Universitaria Konrad Lorenz.

Reese, R. J., Usher, E. L., Bowman, D. C., Norsworthy, L. A., Halstead, J. L., Rowlands, S. R., y Chrisholm, R. R. (2009). Using client feedback in psychotherapy training: An analysis of its influence on supervision and counselor self-efficacy. Training and Education in Professional Psychology, 3(3), 157-168. Recuperado de https://www.researchgate.net/publication/232474369_Using_Client_Feedback_in_Psychotherapy_Training_An_Analysis_of_its_Influence_on_Supervision_and_Counselor_Self-Efficacy

Reiser, R. P., \& Milne, D. (2012). Supervising cognitive-behavioral psychotherapy: Pressing needs, impressing possibilities. Journal of Contemporary Psychotherapy, 42(3), 161-171. doi: 10.1007/s10879-011-9200-6. 
Reiser, R. P., \& Milne, D. L. (2014). A systematic review and reformulation of outcome evaluation in clinical supervision: Applying the fidelity framework. Training and Education in Professional Psychology, 8(3), 149-157. http://dx.doi. org/10.1037/tep0000031.

Reiser, R., y Milne, D. (2017). A CBT formulation of supervisees' narratives about unethical and harmful supervision. The Clinical Supervisor, 36(1), 102-115. http:// www.tandfonline.com/doi/abs/10.1080/07325223.2017.1295895.

Rodolfa, E., Bent, R., Eisman, E., Nelson, P., Rehm, L., \& Ritchie, P. (2005). A cube model for competency development: Implications for psychology educators and regulators. Professional Psychology: Research and Practice, 36(4), 347-354. http://dx.doi.org/10.1037/0735-7028.36.4.347.

Rodolfa, E., Greenberg, S., Hunsley, J., Smith-Zoeller, M., Cox, D., Sammons, M., Caro, C., \& Spivak, H. (2013). A competency model for the practice of psychology. Training and Education in Professional Psychology, 7(2), 71-83. http://dx.doi. org/10.1037/a0032415.

Rodríguez, D., \& Niño, J. A. (2006). Marco comprensivo para el estudio de los procesos de formación de terapeutas. Diversitas, 2(1), 42-54. http://dx.doi. org/10.15332/s1794-9998.2006.0001.03.

Rosenbluth, A., Cruzat-Mandich, C. V., \& Ugarte, M. L. (2016). Metodología para validar un instrumento de evaluación por competencias en estudiantes de psicología. Universitas Psychologica, 15(1), 303-314.

Spence, S. H., Wilson, J., Cavanag, D., Strong, J., \& Worrall, L. (2001). Clinical supervision in four mental health professions: A review of the evidence. Behaviour Change, 18, 135-155.

Tomlin, A. M., Weatherston, D. J., \& Pavkov, T. (2014). Critical components of reflective supervision: Responses from expert supervisors in the field. Infant Mental Health Journal, 35, 70-80. doi: 10.1002/imhj.21420.

Tsong, Y., \& Goodyear, R. K. (2014). Assessing supervision's clinical and multicultural impacts: The supervision outcome scale's psychometric properties. Training and Education in Professional Psychology, 8, 189-195. doi: 10.1037/tep0000049. 
Whalen, T., \& Wendel, G. (2011). New supervision standards: Discussion and justification. In I. Philibert \& S. Amis, Jr. (Eds.), The ACGME 2011 duty hour standards: Enhancing quality of care, supervision, and resident professional development (pp. 39-45). Chicago, IL: ACGME.

Watkins, E., \& Scaturo, D. (2013). Toward an integrative, learning-based model of psychotherapy supervision: Supervisory alliance, educational interventions, and supervisee learning/relearning. Journal of Psychotherapy Intregration, 23, 7595. doi: 10.1037/a0031330.

Wielenska, R. C., \& Oshiro, C. K. B. (2012). FAP group supervision: Reporting educational experiences at the University of São Paulo, Brazil. International Journal of Behavioral Consultation and Therapy, 7(2-3), 177-181. http://dx.doi. org/10.1037/h0100953. 


\section{Anexos}

Tabla A1. Significancia estadística de las comparaciones de las medidas de lápiz y papel

\begin{tabular}{l|c|c|c|c}
\multirow{2}{*}{\begin{tabular}{l} 
Comparación \\
\cline { 2 - 4 }
\end{tabular}} & $\begin{array}{r}\text { Comparaciones entre } \\
\text { el grupo experimental } \\
\text { y el de control }\end{array}$ & \multicolumn{2}{c}{$\begin{array}{c}\text { Comparación entre pretest } \\
\text { y postest por cada grupo }\end{array}$} \\
\cline { 2 - 5 } & Pretest & Postest & Grupo control & Grupo experimental \\
\hline $\begin{array}{l}\text { SAGE } \\
\text { Estructura de supervisión }\end{array}$ & 0,174 & 0,572 & 0,18 & 0,527 \\
\hline $\begin{array}{l}\text { SAGE } \\
\text { Proceso de supervisión }\end{array}$ & 0,267 & 0,792 & 0,18 & 0,527 \\
\hline SOS & 0,393 & 0,114 & 0,564 & 0,827 \\
\hline
\end{tabular}

Tabla A2. Significancia estadística de las comparaciones de la medida de observación SAGE heteroaplicado

\begin{tabular}{l|c|c|c|c}
\multirow{2}{*}{\multicolumn{1}{c|}{ Comparación }} & \multicolumn{2}{c|}{$\begin{array}{c}\text { Comparaciones entre } \\
\text { el grupo experimental } \\
\text { y el de control }\end{array}$} & \multicolumn{2}{c}{$\begin{array}{c}\text { Comparación entre pretest } \\
\text { y postest por cada grupo }\end{array}$} \\
\cline { 2 - 5 } & Pretest & Postest & Grupo control & Grupo experimental \\
\hline $\begin{array}{l}\text { SAGE } \\
\text { Estructura de supervisión }\end{array}$ & 0,269 & 0,52 & 0,549 & 0,45 \\
\hline $\begin{array}{l}\text { SAGE } \\
\text { Proceso de supervisión }\end{array}$ & 0,607 & 0,123 & 0,336 & 0,853 \\
\hline $\begin{array}{l}\text { SAGE } \\
\text { Ciclo de aprendizaje }\end{array}$ & 0,583 & 0,194 & 0,695 & 0,705 \\
\hline
\end{tabular}

